\title{
Role of intestinal brush border peptidases in the simulated digestion of milk proteins
}

Gianluca Picariello ${ }^{1, *}$, Beatriz Miralles ${ }^{2}$, Gianfranco Mamone ${ }^{1}$, Laura Sánchez-Rivera ${ }^{2}$, Isidra Recio $^{2}$, Francesco Addeo ${ }^{3}$, Pasquale Ferranti ${ }^{3}$

${ }^{1}$ Istituto di Scienze dell’Alimentazione- Consiglio Nazionale delle Ricerche, Via Roma 64, 83100 Avellino, Italy.

${ }^{2}$ Instituto de Investigación en Ciencias de la Alimentación (CIAL), CSIC, Nicolás Cabrera 9, 28049 Madrid, Spain

${ }^{3}$ Dipartimento di Agraria, Università di Napoli “Federico II”, Parco Gussone, 80055 Portici (Napoli), Italy

* Corresponding author: Gianluca Picariello, Istituto di Scienze dell’Alimentazione- Consiglio Nazionale delle Ricerche, Via Roma 64, 83100 Avellino, Italy.

Tel. +39 0825 299521; Fax: + 390825781585

Email: picariello@isa.cnr.it 


\section{ABSTRACT}

Scope: This study aimed to assess the impact of the "often neglected" intestinal brush border membranes (BBM) hydrolases on dietary peptides, exploring the possibility that the disintegration of proteins progressed in the small intestine up to a "core" of intrinsically stable oligopeptides, persisting independently on the up-stream breakdown.

Methods and results: Samples of sodium caseinate, skim milk powder and whey protein isolate were submitted to in vitro simulated gastro-pancreatic digestion using two different procedures: i) a simplified model involving the main compartmental specific proteases; ii) a static digestion method based on a frameset of parameters inferred from in vivo. The gastro-duodenal digesta were further hydrolyzed with peptidases from porcine jejunal BBM.

The peptidomes arising from the two digestion models, characterized by combined HPLC and MS techniques, differed to some extent. However, only specific protein domains survived digestion, among which potential bioactive or immunogenic (food allergy) peptides. The degree of hydrolysis after BBM digestion (70-77\%) practically did not differ between the digestion models and significantly increased the degree of hydrolysis after duodenal steps.

Conclusions: Any in vitro digestion model should be supplemented with a jejunal phase to realistically determine the bioaccessibility and bioavailability of dietary peptides.

Keywords: in vitro digestion models; milk proteins; brush border membrane hydrolases; gastrointestinal digestion; degree of hydrolysis. 


\section{Abbreviations:}

BAEE, N- $\alpha$-benzoyl-L-arginine ethyl ester; BBM, brush border membrane; $\mathbf{C N}$, sodium caseinate; DAD, diode array detector; DH, degree of hydrolysis; ESI, electrospray ionization; GI, gastrointestinal; IT, ion trap; LIFT, laser-induced forward transfer; MALDI-TOF, matrix assisted laser desorption ionization-time of flight; $\mathbf{M S}$, mass spectrometry; MS/MS tandem mass spectrometry; RP-HPLC, reversed phase-high performance liquid chromatography; SMP, skim milk powder; WPI, whey protein isolate. 


\section{INTRODUCTION}

The high stability of specific domains of dietary proteins to the harsh conditions present in the gastrointestinal (GI) tract suggests that some peptides are able to cross the gut mucosal barrier and could exert various beneficial effects (bioactive peptides) or, on the contrary, elicit an immune response (food allergy).

Under this standpoint, milk proteins, assumed as a model of dietary proteins, are by far the most extensively studied. According to a large body of literature, the peptides released during GI digestion cover a wide range of bioactive or immunogenic sequences. However, the evidences about the bioactive potential of milk-derived peptides have been almost entirely obtained in conditions sometimes divergent from those occurring along the human GI tract.

A simplified static and multi-phasic in vitro model of the GI digestion, consisting of sequential simulated gastric, duodenal and intestinal (peptidases from human intestinal brush border membrane enzymes) phases, has been previously adapted and applied to identify both the caseinand whey protein-derived peptides particularly resistant to the GI proteolysis [1, 2].

Composition of the digestion fluids, incubation times and proteases-to-proteins ratio of such a simplified model differed in several aspects from those of the in vitro static model of the GI digestion that has been recently harmonized within the frame of the Infogest Cost Action [3]. According to this latter procedure, ion composition and $\mathrm{pH}$ of the digestive juices as well as concentration of the hydrolytic enzymes have been defined on the basis of a set of parameters that has been deduced from in human determinations.

However, many milk-derived peptides that were found to endure proteolysis in simplified conditions corresponded to or in part overlapped those identified as resistant in vivo [4, 5], thus suggesting that specific domains of milk proteins have an intrinsic stability to digestion and could be good candidates to exhibit biological activity. On the other hand, the number and amount of milk-derived peptides that survive the simplified simulated digestion were greatly reduced if compared to other models of the gastro-duodenal digestion, mainly because the process also 
included a final step of degradation with the endo-/exo-peptidases from intestinal brush border membranes (BBM). To date, several studies have demonstrated that some milk-derived peptides surviving the gastro-pancreatic digestion are degraded by the BBM-associated peptidases [2, 6].

The entire intestinal tract is lined by a continuous layer of epithelial enterocytes characterized by apical structures that compose thousands of microvilli (BBM vesicles), which generate a strikingly large membrane surface area. The microvilli protrusions are particularly accentuated in the jejunum, that in an adult individual is $~ 2.5-3.0 \mathrm{~m}$ long. Intestinal BBM vesicles contain at least 12 evolutionarily highly conserved peptidases, within a more than double number of general hydrolases (e.g. glicosidases) [7]. Interestingly, the inventory of the intestinal hydrolases has been significantly extended, even up to an order of magnitude higher, by recent proteomic investigations [8]. Such a body of information highlights the key physiological role played in vivo by the intestinal BBM peptidases for subserving the terminal peptide digestion into short oligopeptides and free amino acids.

Although food-derived oligopeptides could exert biological actions locally, at level of the intestinal lumen, the resistance to intestinal epithelial hydrolases is a prerequisite for the intestinal absorption of peptides and access to the sub-epithelial lymphocytes as well as for systemic distribution. In this sense, in vitro digestion models that include a step of intestinal digestion with BBM peptidases have an already validated physiological consistency, as they supported, for instance, the discovery of wheat prolamin-derived peptides that elicit celiac disease in vivo [9-11]. In spite of its importance to assess the bioaccessibility and bioavailability of potential bioactive or immunogenic peptides, the intestinal phase of digestion with BBM peptidases has been omitted in the majority of the in vitro digestion models developed so far, probably also because, differently from the other digestion compartments, a consensus about the parameters relevant to the simulated jejunal digestion remains far from being established.

In this work we analysed comparatively the digesta of three milk protein samples, namely caseinate (CN), skim milk powder (SMP) and whey protein isolate (WPI), arising from two digestion 
procedures: i) the original "simplified” model [1,2], that was adapted to fit the conditions by Shan et al. [9]; ii) a model integrated with parameters inferred from in vivo determinations, thereinafter referred to as "integrated" model, that used conditions very similar to those of the recently harmonized static model [3]. Both the procedures were completed with an additional step of simulated intestinal degradation with BBM enzymes. In the perspective of the definition of physiologically relevant parameters to set up comprehensive in vitro models of the GI digestion, this study investigated the impact of the intestinal BBM hydrolases on the production and evolution of dietary proteins and peptides.

\section{MATERIALS AND METHODS}

\subsection{Powdered milk proteins and chemicals}

Samples of CN, SMP and WPI powders were provided by the Fonterra Dairy Co. via the Riddett Institute (New Zealand). Porcine pancreatic enzymes (pepsin >2,500 U/mg, trypsin >10,000 BAEE $\mathrm{U} / \mathrm{mg}$, chymotrypsin $>40 \mathrm{U} / \mathrm{mg}$ and elastase $>4 \mathrm{U} / \mathrm{mg}$ ), solvents and chemicals, including phosphatidylcholine, bile salts/acids (taurocholate/glicodeoxycholic acid) and MALDI matrices were purchased from Sigma (St. Louis, MO, USA). Carboxypeptidase A was from Roche (Mannheim, Germany).

\subsection{Simplified in vitro gastro-duodenal digestion}

Gastro-duodenal digestion of CN, SMP and WPI was substantially performed as previously described [1, 2]. Briefly, CN, SMP and WPI were dissolved in 5\% formic acid at the concentration of $2 \mathrm{mg} / \mathrm{mL}$ and incubated at $37^{\circ} \mathrm{C}$ with pepsin (1:100 enzyme/protein ratio, w/w) for $60 \mathrm{~min}$, under low magnetic stirring. Before pancreatic digestion, the samples were evaporated and reconstituted twice with deionised water. Peptic digests were redissolved in $0.1 \mathrm{M}$ sodium phosphate buffer (pH 7.0) supplemented with trypsin (1:100, w/w), chymotrypsin (1:100, w/w), elastase (1:500, w/w) and carboxypeptidase A (1:100, w/w) and incubated $1 \mathrm{~h}$ at $37^{\circ} \mathrm{C}$. The reaction was stopped by heating for $5 \mathrm{~min}$ in a boiling water bath. The aliquots for the subsequent intestinal reactions were directly 
supplemented with BBM, omitting the 5 min boiling. As checked by RP-HPLC, heating induced no significant alteration of the peptide patterns.

\subsection{Integrated gastro-duodenal static digestion model}

The “integrated" procedure was developed during a "pre-harmonization" stage of the static in vitro digestion model recently standardized in the framework of the Infogest project [3]. CN, SMP and WPI were digested under conditions very close to those of the harmonized model [3], in line with the procedure described by Sanchez-Rivera et al. [12]. The oral phase of digestion was omitted, due to the absence of starchy matrices in the milk protein samples. Briefly, CN, SMP and WPI were dissolved (13 mg/mL of protein) in simulated gastric fluid (35 mM NaCl) acidified with $\mathrm{HCl}$ at $\mathrm{pH}$ 2 and preheated at $37^{\circ} \mathrm{C}$. Gastric digestion was carried out with porcine pepsin at an enzyme/substrate ratio of $1: 20 \mathrm{w} / \mathrm{w}(182 \mathrm{U} / \mathrm{mg})$ at $37^{\circ} \mathrm{C}$ during $1 \mathrm{~h}$, in the presence of phosphatidylcholine vesicles and $2.5 \mathrm{mM} \mathrm{CaCl}_{2}$. Peptic digestion was stopped by adjusting the $\mathrm{pH}$ of the reaction mixture to 7.0 with $\mathrm{NaOH}$. The in vitro duodenal digestion was carried out on the products from stomach digestion supplemented with $1 \mathrm{M} \mathrm{CaCl}_{2}, 0.25 \mathrm{M}$ Bis-Tris pH 6.5 and 0.125 M equimolar mixture of sodium taurocholate and glicodeoxycholic acid. The final concentrations within the mixture were $3.9 \mathrm{mg} / \mathrm{mL}$ of protein, $7.4 \mathrm{mM}$ of bile salts, $7.6 \mathrm{mM}$ of $\mathrm{CaCl}_{2}$ and $20.3 \mathrm{mM}$ of Bis-Tris. Trypsin (40 U/mg protein), chymotrypsin (0.5 U/mg protein), porcine pancreatic lipase (28.9 U/mg protein) and colipase (enzyme/substrate ratio 1/895, w/w) were diluted in $35 \mathrm{mM} \mathrm{NaCl,}$ adjusted to $\mathrm{pH} 7$ and incorporated into the reaction mixture.

\subsection{Preparation of porcine intestinal BBM}

Surgical specimens of intestinal jejunum (cut in $\sim 4 \mathrm{~cm}^{2}$ pieces) were obtained from an adult pig within a few minutes after slaughtering in a local animal farm, immediately frozen on dry ice and stored at $-80^{\circ} \mathrm{C}$ until used. Anatomic identification of the jejunal section was confirmed by microscopic inspection of the tissue. Porcine intestinal BBM vesicles were purified according to the method by Shirazi-Beechey et al. [13]. To this end, specimens were thawed in ice-cold $50 \mathrm{mM}$ mannitol, 2mM Tris-HCl, $\mathrm{pH}$ 7.1. Cells were removed from the connective tissue by using a 
Vibromixer (model E-1, Alpha Laval, UK) at max speed $2 \times 1$ min. The cell suspension was homogenised with an Ultraturrax T 25 (IKA, Works, Inc., USA) and diluted with $\mathrm{MgCl}_{2}$ to a final concentration of $10 \mathrm{mM}$. The suspension was stirred $20 \mathrm{~min}$ at $0^{\circ} \mathrm{C}$ and then cell debris, basolateral, membranes, nuclei, and mitochondria were eliminated by centrifugating at $3000 \times \mathrm{g}, 15 \mathrm{~min}$ at $4^{\circ} \mathrm{C}$. The supernatant was centrifuged at $30,000 \times \mathrm{g}, 30 \mathrm{~min}$ at $4^{\circ} \mathrm{C}$. The pellet was resuspended in $6 \mathrm{~mL}$ of $300 \mathrm{mM}$ mannitol, $0.1 \mathrm{mM} \mathrm{MgSO}$, and $2 \mathrm{mM}$ Tris $\mathrm{pH} 7.4$ to obtain a clear protein solution that was passed several times through a 27-gauge needle; the brush-border vesicles were stored in aliquots at $-80^{\circ} \mathrm{C}$. Shortly before use, the vesicles were thawed on ice and washed with the sodium phosphate buffer. Peptidase activity (55 $\mu \mathrm{U} / \mu \mathrm{L})$ was determined by time-monitoring the hydrolysis of known amounts of a standard peptide (angiotensin-I) in $0.1 \mathrm{M}$ Tris $\mathrm{HCl} \mathrm{pH} 7.0$ buffer with BBM extracts using reversed phase-high performance liquid chromatography (RP-HPLC), up to the complete disappearance of detectable peptides and to constant values of free Phe and Tyr (monitored at $\lambda=280 \mathrm{~nm}$ ) [14]. One unit is defined as the hydrolysis of $1 \mu \mathrm{mol}$ peptide bond/min [10].

\subsection{Simulated intestinal digestion with porcine BBM}

The peptide digests arising from gastro-duodenal digestion were two-fold diluted with $0.1 \mathrm{M}$ sodium phosphate buffer $\mathrm{pH}$ 7.2, supplemented with $100 \mu \mathrm{U} B \mathrm{BM} / 100 \mu$ g peptides and incubated at $37^{\circ} \mathrm{C}$ up to $6 \mathrm{~h}$. The peptidase activity-to-substrate ratio was selected according to previous literature studies [14]. The amount of the peptide substrate was estimated according to the original protein material, hence containing at this stage free amino acids too. Aliquots were picked up and analyzed at intermediate incubation times. The entire digestion procedures were performed in triplicate.

\subsection{Determination of the degree of hydrolysis}

The degree of hydrolysis (DH) was determined with the 2,4,6-trinitrobenzene sulfonic acid (TNBS) method [15], according to Hermanson (1996) [16]. Briefly, $5.0 \mu \mathrm{g}$ of the sample proteins (CN, SMP and WPI) and the corresponding digesta were diluted in $0.5 \mathrm{~mL}$ of $0.1 \mathrm{M}$ sodium bicarbonate, $\mathrm{pH}$ 
8.5. To each sample, $0.25 \mathrm{~mL}$ of $0.01 \%$ TNBS (Sigma) was added and incubated at $37^{\circ} \mathrm{C}$ for $2 \mathrm{~h}$. The sampling volumes were calculated taking into account the opportune dilution factors at the several digestion steps. The reaction was stopped with $0.25 \mathrm{~mL}$ of $10 \%$ SDS (w/v) followed by $0.125 \mathrm{~mL}$ of $1 \mathrm{~N} \mathrm{HCl}$. The absorbance of the chromogenic derivatives was measured at $335 \mathrm{~nm}$ using an Ultrospec 2100-Pro (Amersham Biosciences, Uppsala, Sweden), against a blank solution prepared as above except for the addition of the protein material. The absorbance values were referred to those obtained by assaying $5.0 \mu \mathrm{g}$ of sample proteins undigested $(\mathrm{DH}=0 \%)$ or undergone complete hydrolysis performed with $6 \mathrm{~N} \mathrm{HCl}$ at $110^{\circ} \mathrm{C}$ for $24 \mathrm{~h}(\mathrm{DH}=100 \%)$ in vacuum tubes (Thermo Scientific, Rockford, IL, USA). All samples were assayed in triplicate and absorbance values were averaged. $\mathrm{DH}=\mathrm{h} / \mathrm{h}_{\text {tot }} * 100$, was measured as the percentage of cleaved (h) with respect to the total number of peptide bonds $\left(\mathrm{h}_{\text {tot }}\right)$, this latter being separately determined after acid hydrolysis of the milk protein substrates.

\subsection{RP-HPLC analysis}

The course of simulated gastro-duodenal-intestinal hydrolysis was monitored by RP-HPLC using a HP1100 modular system (Agilent, Palo Alto, CA, USA) equipped with a Jupiter ${ }^{\circledR}$ C18 RP column, $2.0 \mathrm{~mm}$ i.d. $\times 250 \mathrm{~mm}$, 4 $\mu \mathrm{m}$ particle diameter (Phenomenex, Torrance, CA, USA). Peptide separation (25 $\mu$ g for each analysis) was carried out with a 5-60\% gradient of solvent B over 60 min at a flow rate of $0.2 \mathrm{~mL} / \mathrm{min}$, after $5 \mathrm{~min}$ of isocratic elution at $5 \% \mathrm{~B}$. Solvent A was $0.1 \%$ TFA (v/v) in water; solvent B was $0.1 \%$ TFA (v/v) in acetonitrile. The column was equilibrated at 5\% B. The column effluent was monitored by UV detection (220 and $280 \mathrm{~nm}$ ) using DAD. The identification of free Phe, Tyr and Trp was confirmed by separate injections of the standard amino acids (Sigma). RP-HPLC analyses were also performed to assess the reproducibility of the digestion procedures.

\subsection{RP-HPLC-MS/MS analysis}

RP-HPLC-MS/MS analyses of peptide digesta were carried out using an Agilent 1100 HPLC System (Agilent Technologies, Palo Alto, CA, USA) coupled to an Esquire 3000 quadrupole IT 
(Bruker Daltonik GmbH, Bremen, Germany) equipped with an ESI source. Peptide separations were performed with a Mediterranea Sea18 $150 \mathrm{~mm} \times 2.1 \mathrm{~mm}$ column (Teknokroma, Barcelona, Spain), applying a linear gradient from 0 to $45 \%$ of solvent B (TFA $0.027 \%$ in acetonitrile) in 120 min, after 5 min of isocratic elution at 0\%. Solvent A was $0.037 \%$ TFA in water. The injection volume was $50 \mu \mathrm{L}$ and the flow rate $0.2 \mathrm{~mL} / \mathrm{min}$. The ESI capillary voltage was $4 \mathrm{kV}$. Spectra were recorded over the $250-1500 \mathrm{~m} / \mathrm{z}$ range. In these analyses, the target mass was set at $750 \mathrm{~m} / \mathrm{z}$. Data were processed using the Data Analysis ${ }^{\mathrm{TM}}$ software (Ver. 4.0; Bruker Daltoniks). Peptides were identified from the MS(n) spectra with the aid of the BioTools (Ver 3.2) software (Bruker Daltoniks), searching an homemade database with bovine caseins, most abundant whey proteins and main genetic variants thereof. No cleavage specificity was indicated. Identifications were considered successful when the measured molecular weight (MW) corresponded to the expected value and a sequence of at least three (two for low sized peptides, $\mathrm{MW}<600$ ) consecutive b- or $\mathrm{y}$ fragment ions occurred in the spectrum. Entries occurring in both of two replicate analyses, with a score higher than the threshold value corresponding to the identity $(\mathrm{p}<0.05)$, were included into the data set. Peptide identification was validated by manual inspection of the MS/MS spectra.

\subsection{MALDI-TOF and TOF/TOF MS}

Prior to MALDI analysis peptide mixtures were desalted using $\mathrm{C}_{18}$ ZipTip micro-columns (Millipore, Watford, UK). MALDI-TOF MS and LIFT ${ }^{\mathrm{TM}} \mathrm{MS} / \mathrm{MS}$ spectra were acquired using an Autoflex speed $^{\mathrm{TM}}$ Instrument (Bruker Daltonik GmbH) operated in the positive reflector ion mode. In MS mode the 600-4500 m/z range was explored. Precursor ions for LIFT MS/MS fragmentation were automatically selected when they overcame a threshold of $4 \times 10^{3}$ ion counts at a laser power in the range 30-55\%. $\alpha$-cyano-4-hydroxycinnamic acid (saturated solution in 30\% acetonitrile/0.1\% TFA, v/v) and 2,5-dihydroxybenzoic acid (10 mg/mL in 50\% acetonitrile/0.1\% TFA) were used as the matrices. Matrix $(0.5 \mu \mathrm{L})$ and peptide solutions $(0.5 \mu \mathrm{L})$ were mixed directly onto the Anchor Chip $^{\text {TM }}$ MALDI target (Bruker Daltonik GmbH) and air-dried. Spectra were externally calibrated using a mixture of standard low-molecular mass peptides (Bruker). 


\section{RESULTS AND DISCUSSION}

\subsection{Monitoring of digestion kinetics of milk proteins by RP-HPLC}

The digestion kinetics of milk proteins was monitored by RP-HPLC. In line with a wealth of literature evidences, casein degradation started early as compared to the whey proteins. As expected $[1,17]$, CN were promptly degraded by pepsin whereas whey proteins, mostly $\beta$-lactoglobulin ( $\beta$ Lg), were still in detectable amounts in SMP and WPI after 1-h incubation with pepsin in a simulated gastric environment. The outcomes of the peptic digestion were substantially similar regardless of the model between the two tested models (data not shown).

After simulated duodenal hydrolysis, intact milk proteins were no longer detectable, while the HPLC chromatograms exhibited complex patterns of small- and medium-sized peptides (Fig.1, blue lines).

The hydrolytic enzymes of pancreatin in the integrated model were employed at a higher proteases-to-milk proteins ratio and included bile salts for stabilizing emulsions. Thus, the duodenal phase of the integrated model was expected to hydrolyze milk proteins more extensively, compared to the simplified one. Even so, free tryptophan (HPLC peak at $24 \mathrm{~min}$, identified by DAD) was much more abundant in the digesta arising from the simplified model, which included elastase and carboxypeptidase $\mathrm{A}$ in the proteolytic system, in addition to trypsin and chymotrypsin. The peptide patterns of the duodenal digesta appeared rather different between the two models, reflecting the substantial difference of the digestion conditions (Fig. 1).

So far, no specific indications are available about a physiological-like use of BBM jejunal enzymes. For this reason, we adopted conditions and levels of enzyme activities in line with those procedures that have already demonstrated a certain in vitro-in vivo consistency $[10,14]$.

The peptides arising from all the milk protein substrates incubated at final step with BBM were intensely degraded, independently on the up-stream model of gastro-duodenal digestion. Indeed, at the end of both the simulated intestinal digestion procedures, only a low amount of 
peptides was detected by HPLC (Fig. 1, red lines) while the intense peak of free aromatic amino acids, especially tryptophan, dominated all the chromatographic profiles. The level of free tryptophan can be exploited as an indicative parameter of the hydrolysis degree of food proteins [18]. Free tryptophan after digestion with BBM, deduced from area under the peaks, had comparable values for the same protein substrates digested according the two different methods, thereby indicating a similar degree of hydrolysis achieved by BBM peptidases. The digestion procedures produced reproducible peptide patterns, as assessed by RP-HPLC analysis of three replicates for each sample (not shown).

\subsection{Degree of protein hydrolysis}

DH values obtained at the several stages of digestion are reported in both the table and graphical layout in Figure 2.

In spite of the lower pepsin-to-protein ratio (1:100 vs. 1:20), the gastric digestion with the simplified model split milk proteins into medium- and large-sized (poly)peptides more efficiently, due to the protective effect of phospholipid vesicles used in the integrated model [19-21]. The hydrolysis progression was largely reverted after duodenal digestion. Due to the higher relative amount of hydrolytic enzymes present in the pancreatin preparation (used at an approximate ratio 1:10 w:w) as well as to the emulsifying properties of bile salts that remarkably promote proteolysis [22], DH was 55.3-59.3\% for the integrated model, clearly higher than $34.5-41.1 \%$ for the simplified model (values are means of three replicate determinations). These figures contrast to some extent with the higher amounts of free aromatic amino acids detected by HPLC in the digesta obtained with the simplified model. However, this apparent discrepancy has to be considered as the result of different proteolytic systems and dissimilar cleavage specificity at the level of the aromatic amino acids, which is testified by a prominent pepsin-chymotrypsin and carboxypeptidase A sequential action in the simplified model. The occurrence of longer peptides in the duodenal pools obtained with the integrated model is most likely due to a less effective primary proteolysis that occurred in the gastric phase (Fig. 2). The DH values measured after gastro-duodenal digestion with 
the integrated model are in good agreement with those previously determined on similar protein substrates, using the $o$-phthalaldehyde (OPA) method [23].

At the end of the intestinal phase DH values were ultimately equalized, i.e. $70.7-77.4 \%$ vs. 70.677.5\%. In particular, $\mathrm{DH}$ of $\mathrm{CN}$ was practically identical between the two digestion procedures. Only minor differences between $\mathrm{DH}$ values were measured for the whey protein-containing samples (SMP and WPI). In other terms, the final peptide degradation with BBM enzymes had a "levelling" effect regardless of the digestion model. Roughly three-fourths of all the peptide bonds of milk proteins were cleaved, contributing to intrigue the still unaddressed issue about the actual survival of medium- and large-sized peptides at concentrations that could be physiologically relevant.

The outstandingly high DH values determined for the three protein substrates are consistent with the HPLC analysis which showed the progressive appearance of intense peaks of free aromatic amino acids (i.e. tryptophan, tyrosine and phenylalanine) and the survival of very low amounts of longer peptides at the end of digestion. These figures of DH did not conflict with the relatively high MS-based coverage of $\beta$-casein and $\beta$-Lg (see below), since $\mathrm{DH}$ and protein coverage are not correlated. Indeed, a given peptide can derive from a very low percentage of the total parent protein.

\subsection{Comprehensive characterization of digesta by MS-based techniques}

To identify the most resistant domains of the milk-proteins, as well as to infer information about the impact of relevant operative parameters on proteolysis, the digesta derived from the complete gastro-duodenal-intestinal BBM degradation were characterized by complementary MS-based techniques. More in details, peptides resulting from the digestion of CN, SMP and WPI with both the models were analyzed by RP-HPLC-ESI-IT-MS/MS and MALDI-TOF-MS also followed by LIFT-TOF/TOF-MS/MS. Confirming previous outcomes [1], free aromatic amino acids (primarily tryptophan) were largely dominant in all the digesta. The intense peaks of Tyr, Phe and Trp were assigned by DAD monitoring of the RP-HPLC-MS analysis as they missed in the total ion current (TIC) chromatograms (detection in the $\mathrm{m} / \mathrm{z}$ range 250-1500). Consistently, the full scan TIC-MS chromatograms showed only relatively low-intensity ions, confirming the occurrence of very low 
peptide amounts in the digesta (not shown). The chromatograms of SMP digesta did not significantly differed from those obtained for $\mathrm{CN}$ and, for some minor traits, from WPI samples (see also below).

TIC profiles of CN digested according the two models appeared quite different while the patterns of WPI digesta were more similar to each other. Whey proteins are characterized by a defined folding sustained by disulfide bridges and by fat binding properties (especially $\beta$-Lg) while caseins are substantially rheomorphic proteins with extremely open and flexible conformation. Thus, this somehow unexpected finding suggests that digestion conditions affected casein degradation at larger extent than whey proteins.

The task of peptide identification was not straightforward due to the presence of a multitude of lowabundant peptides generated by random or not predictable cleavage along the backbone of proteins. Partial exo-peptidase hydrolysis produced a series of progressively shortened peptides differing from each other by a single amino acid residue. The milk-derived peptides survived survived at a low amounts and the resolution of the HPLC separation decreased when higher amounts of digesta were injected, due to the presence of interfering compounds. Approximately from 50-75 peptides were identified in the digesta by software-assisted MS data mining and manual inspection of the ESI-IT MS and MS(n) spectra, also aided by previous results [1, 24]. The information deriving from the MALDI-based MS spectra was as much valuable, especially for the identification of relatively large peptides.

The TIC chromatograms of the digesta arising from the integrated model contained out-of-scale peaks at high retention times not detected by UV (not shown), due to co-eluting phospholipids and bile salts, omitted in the simplified procedure. Gross comparison of the relative peptides-to-lipids peak ratio confirmed that only low amounts of peptides survived digestion. The largest part of peptides in the $\mathrm{CN}$ digests was derived from $\beta$-casein and $\alpha_{\mathrm{s} 1}$-casein (70\% and $30 \%$, respectively). The vast majority of peptides of WPI digesta arose from $\beta$-Lg. Many of the identified peptides arising from the simplified digestion model corresponded to those already described [1], as the 
hydrolysis conditions were practically the same, except for the origin of intestinal BBM vesicles that were porcine in the current study instead of human.

The Figure 3 depicts the comparison between $\beta$ - and $\alpha_{s 1}$-casein peptides in the CN digesta obtained from the two digestion models after BBM hydrolysis. The peptides were identified by complementary ESI and MALDI MS-based techniques. The two digesta share only a few common $\beta$-casein peptides. This observation confirmed that the hydrolysis conditions have a key impact on the nature of the produced protein fragments. Nevertheless, a great percentage of peptides in both digesta arose from only three $\beta$-casein regions, namely 74-92, 130-138 and 184-209, subsequently shortened by the action of duodenal carboxypeptidase A and BBM exo-peptidases. Thus, it partly holds the hypothesis that only a few specific casein domains are intrinsically stable to proteolysis.

Our analysis also revealed that the pattern of the $\beta$-casein peptides from the integrated model was similar to that observed in vivo, by analysis of the intestinal aspirates [5]. On the other hand, peptides in the in vivo digesta were much more numerous and abundant, arguably because at least part of them had not reached intestinal apical membranes yet. Interestingly, the stable regions of $\beta$ casein are precursors of sequences described as bioactive peptides: the region 74-92 harbours IPP and VPP described as digestion resistant antihypertensive tri-peptides [25], which are also able to translocate cell models of the intestinal epithelium [26]; similarly, $\beta$-casein f193-209 contained in the 184-209 domain appears to partly survive digestion and to pass across Caco-2 cell monolayers [27]; $\beta$-casein f130-138 encrypts the angiotensin-converting enzyme I-inhibitor penta-peptide HLPLP that is resistant to digestive enzymes and, once absorbe, is processed by blood proteases, releasing still active fragments [28]. Peptides belonging to the strategic zone of the $\beta$-casomorphins (region 60-70) were detected at trace amounts especially with the integrated model.

It remains to establish if the actual bioaccessibility of the potential bioactive peptides, strongly reduced by BBM peptidases, is high enough to induce relevant bioactive effects in vivo. 
The comparison between $\alpha_{s 1}$-casein derived peptides (Fig. 3, right panel) supports the conclusion that only a few intrinsically stable peptide regions survive the proteolytic breakdown of the whole process, as there is a common domain in both $\mathrm{CN}$ digests where the majority of the identified peptides belonged, i. e. the 180-193 region. Interestingly, several processed forms of the phosphorylated $\alpha_{\mathrm{s} 1}$-casein f104-119 were identified in the $\mathrm{CN}$ digested according with the simplified model. Only one peptide from $\kappa$-f152-156 and one from $\alpha_{\mathrm{s} 2}$-casein (phosphorylated f138-146) were identified in the digesta following the simplified model.

Comparison of the peptides released from $\beta$-Lg in the WPI digesta is summarized in Figure 4. As already emphasized for the UV-based detection, peptide digesta of whey proteins are similar to each other if compared to casein-containing samples. Indeed, in both cases, most of the $\beta$-Lg peptides derived from three protein regions, variously trimmed at the $\mathrm{N}$ - and C-terminus, i.e. the 4060, 125-138 and 149-162 (protein C-terminal) regions, the latter containing Cys 60, linked through a disulfide bridge to Cys 66 of the 61-70 domain. The intact disulfide cross-linked peptides were identified by MALDI-TOF MS (not shown). Importantly, all of the three most represented domains have been described as the potential IgE-binding immunoreactive epitopes of $\beta$-Lg [29]. Thus, although this result should not be considered a general trait, the (partial) resistance to digestion in this case appears a pre-requisite for the immunogenic activity. It has to be also underlined that in general the MS detectability of the peptides is not correlated to their actual quantity in the digesta. However, information about the peptide abundances can be inferred by the relative peak intensities along the UV chromatograms (after expansion along the Y-axis). The UV detection also confirmed that $\beta$-Lg peptides arising from the 125-138 and 40-60 regions were the most abundant.

Only 5 and 3 short peptides from $\alpha$-lactalbumin ( $\alpha$-La) were identified, in the digesta of the simplified and integrated models, respectively, one of which, $\alpha$-La f114-117, shared by the two digesta. Three $\alpha$-La peptides identified in the digesta from the simplified model were highly acidic (region 80-90, sequence FLDDDLTDDIM), indicating that, as observed for $\beta$-Lg (several peptides 
from the 125-138 region), very acidic sequences are relatively resistant to digestion and could be candidates to be immunogenic in vivo, also because they are spatially located on the external surface of the native IgE-binding globular whey proteins. Notably, the $\alpha$-La f80-90 is practically the unique region of $\alpha$-La found at significant amount in human intestinal aspirates during milk digestion [5]. Minor amounts of high molecular weight peptides ( $\mathrm{m} / \mathrm{z}$ 3500-3800), previously assigned to multiply S-S cross-linked $\alpha$-La peptides [1], were also detected by MALDI-TOF MS. SMP contains both caseins and whey proteins, in the ordinary ratio of bovine milk. Thus, HPLC and HPLC-MS(n) patterns of peptides occurring in the SMP digesta roughly reflected the ratio of casein to whey proteins, of bovine milk (nearly $80 \%$ casein and 20\% whey proteins). SMP, in fact, contained most of the casein-derived peptides identified in $\mathrm{CN}$ and minor amounts of the $\beta$-Lg peptides identified in WPI (not shown). This result indicates that the digestion of caseins and whey proteins in mixture was not appreciably affected by possible mutual interactions.

\section{CONCLUSIONS}

The harmonization of the in vitro digestion models and the definition of experimental protocols that rely on in vivo inferred parameters represent significant advances towards our knowledge about the fate of dietary proteins in the GI tract and their potential impact on human physiology. Relying on a series of parameters determined in human, the integrated model and even more the standardized one [3], are expected to provide more strict physiological consistency than the simplified model. Clearly, the in vitro-in vivo correspondence of the outcomes is the criterion that should drive the choice of a simulated digestion model. On the other hand, while the physiological consistency of any in vitro digestion model remains to be convincingly validated, the simplified model has some technical advantages because it is easy to be performed and in the duodenal step it makes use of lower amounts of pooled purified enzymes at high specific activity if compared to pancreatin. The digesta from the simplified model do not contain the bulk of interfering species (e.g. pancreatic enzymes, phospholipids, bile salts) that complicate the identification of peptides and proteins arising from more elaborated protocols [23]. Thus, depending on the research purposes and on the 
food matrix, selected parameters of the simplified protocol can be included in procedures of simulated digestion in order to balance simplicity and consistency [3].

As a major result, the outcomes of this study point out the need of including a phase of peptide degradation with BBM enzymes within any in vitro digestion model, to mimic the final digestion occurring in vivo in the jejunum. Intestinal peptidases contribute to heavily hydrolyze internally and "gnaw" the peptides released by the gastro-duodenal breakdown up to a "core” of (oligo)peptides, these last resistant to a further hydrolytic cleavage. The striking increase in the degree of hydrolysis induced by BBM enzymes up to a value that is ultimately independent on the up-stream production, cannot be neglected when the in vitro determination of bioaccessibility and, subsequently, bioavailability of dietary peptides is required. Obviously, dedicated investigations are still needed to define the appropriate operative parameters with true physiological significance, for mimicking the intestinal digestion. In this sense, it is expected that the intestinal mucus also plays a key role as a physicochemical barrier able to delay (or impede) peptides and peptidases to come across.

The potential capability of nutrients to transduce nutritional information to the rest of the body by stimulating intestinal receptors - an issue someway related to the possible incomplete BBMmediated hydrolysis and, hence, to the actual bioaccessibility of dietary peptides - launches a further research challenge that is emerging as the "gut chemosensing" field [30].

\section{AKNOWLEDGEMENT}

The research was in part supported by the EU Infogest COST (European Cooperation in Science and Technology) Action FA 1005 'Improving health properties of food by sharing our knowledge on the digestive process' by funding a short scientific mission of G. P. in the laboratories at CIALCSIC (Madrid, Spain).

The Authors declared no conflict of interest. 


\section{REFERENCES}

[1] Picariello, G., Ferranti, P., Fierro, O., Mamone, G. et al., Peptides surviving the simulated gastrointestinal digestion of milk proteins: biological and toxicological implications. $J$. Chromatogr. B Analyt. Technol. Biomed. Life Sci. 2010, 878, 295-308.

[2] Picariello, G. Iacomino, G., Mamone, G., Ferranti, P. et al., Transport across Caco-2 monolayers of peptides arising from in vitro digestion of bovine milk proteins. Food Chem. 2013, 139, 203-212. [3] Minekus, M., Alminger, M., Alvito, P., Balance, S. et al., A standardised static in-vitro digestion method suitable for food - an international consensus. Food Funct. 2014, 5, 1113-1124.

[4] Bouzerzour, K., Morgan, F., Cuinet, I., Bonhomme, C. et al., In vivo digestion of infant formula in piglets: protein digestion kinetics and release of bioactive peptides. Br. J. Nutr. 2012, 108, 21052114.

[5] Boutrou, R., Gaudichon, C., Dupont, D., Jardin, J. et al., Sequential release of milk proteinderived bioactive peptides in the jejunum in healthy humans. Am. J. Clin. Nutr. 2013, 97, 13141323.

[6] Quirós, A., Dávalos, A., Lasunción, M. A., Ramos, M., Recio, I., Bioavailability of the antihypertensive peptide LHLPLP. Transepithelial flux of HLPLP. Int. Dairy J. 2008, 18, 279-286.

[7] Holmes, R., Lobley, R. W., Intestinal brush border revisited. Gut 1989, 30, 1667-1678.

[8] McConnell, R. E., Benesh, A. E., Mao, S., Tabb, D. L., Tyska, M. J. Proteomic analysis of the enterocyte brush border. Am. J. Physiol. Gastrointest. Liver Physiol. 2011, 300, G914-26.

[9] Shan, L., Molberg, Ø., Parrot, I., Hausch, F. et al., Structural basis for gluten intolerance in celiac sprue. Science. 2002, 297, 2275-2279.

[10] Hausch, F., Shan, L., Santiago, N. A., Gray, G. M., Khosla, C. Intestinal digestive resistance of immunodominant gliadin peptides. Am. J. Physiol. Gastrointest. Liver Physiol. 2002, 283, G996G1003.

[11] Mamone, G., Ferranti, P., Rossi, M., Roepstorff, P. et al., Identification of a peptide from alpha-gliadin resistant to digestive enzymes: implications for celiac disease. J. Chromatogr. $B$ Analyt. Technol. Biomed. Life Sci. 2007, 855, 236-241.

[12] Sánchez-Rivera, L., Diezhandino, I., Gómez-Ruiz, J. Á., Fresno, J. M. et al., Peptidomic study of Spanish blue cheese (Valdeón) and changes after simulated gastrointestinal digestion. Electrophoresis 2014, 35, 1627-1636.

[13] Shirazi-Beechey, S. P., Davies, A. G., Tebbutt, K., Dyer, J. et al., Preparation and properties of brush-border membrane vesicles from human small intestine. Gastroenterology 1990, 98, 676-685.

[14] Piper, J. L., Gray, G. M., Khosla, C., Effect of prolyl endopeptidase on digestive-resistant gliadin peptides in vivo. J. Pharmacol. Exp. Ther. 2004, 311, 213-219. 
[15] Adler-Nissen, J., Determination of the Degree of Hydrolysis of Food Protein Hydrolysates by Trinitrobenzenesulfonic Acid. J. Agric. Food Chem. 1979, 27, 1256-1262.

[16] Hermanson, G. T., in: Bioconjugate Techniques, Academic Press, San Diego, CA, USA 1996, pp. 112-113.

[17] Kitabatake, N., Kinekawa Y.-I., Digestibility of Bovine Milk Whey Protein and $\beta$ Lactoglobulin in Vitro and in Vivo. J. Agric. Food Chem. 1998, 46, 4917-4923.

[18] Nielsen, P. M., Petersen, M., Dambmann, C. Improved Method for Determining Food Protein Degree of Hydrolysis. J. Food Sci. 2001, 66, 642-646.

[19] Moreno, F. J., Mackie, A. R., Mills, C. E. N., Phospholipid interactions protect the milk allergen alpha-lactalbumin from proteolysis during in vitro digestion. J. Agric. Food Chem. 2005, 53, 9810-9816.

[20] Mandalari, G., Mackie, A. M., Rigby, N. M., Wickham, M. S., Mills, C. E. N., Physiological phosphatidylcholine protects bovine beta-lactoglobulin from simulated gastrointestinal proteolysis. Mol. Nutr. Food Res. 2009, 53, S131-139.

[21] Berecz, B., Mills, C. E. N., Parádi, I., Láng, F. et al., Stability of sunflower 2S albumins and LTP to physiologically relevant in vitro gastrointestinal digestion. Food Chem. 2013, 138, 23742381.

[22] Gass, J., Vora, H., Hofmann, A. F., Gray, G. M., Khosla, C., Enhancement of dietary protein digestion by conjugated bile acids. Gastroenterology 2007, 133, 16-23.

[23] Kopf-Bolanz, K. A., Schwander, F., Gijs, M., Vergères, G. et al. Validation of an in vitro digestive system for studying macronutrient decomposition in humans. J. Nutr. 2012, 142, 245-250.

[24] Moreno, F. J., Quintanilla-López, J. E., Lebrón-Aguilar, R., Olano, A., Sanz, M. L., Mass spectrometric characterization of glycated beta-lactoglobulin peptides derived from galactooligosaccharides surviving the in vitro gastrointestinal digestion. J. Am. Soc. Mass. Spectrom. 2008, 19, 927-937.

[25] Ohsawa, K., Satsu, H., Ohki, K., Enjoh, M., Takano, T., Shimizu, M. Producibility and digestibility of antihypertensive beta-casein tripeptides, Val-Pro-Pro and Ile-Pro-Pro, in the gastrointestinal tract: analyses using an in vitro model of mammalian gastrointestinal digestion. $J$. Agric. Food Chem. 2008, 56, 854-858.

[26] Foltz, M., Cerstiaens, A., van Meensel, A., Mols, R., van der Pijl, P. C., Duchateau, G. S., Augustijns, P. The angiotensin converting enzyme inhibitory tripeptides Ile-Pro-Pro and Val-Pro- 
Pro show increasing permeabilities with increasing physiological relevance of absorption models. Peptides 2008, 29, 1312-1320.

[27] Regazzo, D., Mollé, D., Gabai, G., Tomé, D. et al. The (193-209) 17-residues peptide of bovine $\beta$-casein is transported through Caco-2 monolayer. Mol. Nutr. Food Res. 2010, 54, 14281435.

[28] Sánchez-Rivera, L., Ares, I., Miralles, B., Gómez-Ruiz, J. et al. Bioavailability and Kinetics of the Antihypertensive Casein-Derived Peptide HLPLP in Rats. J. Agric. Food Chem. 2014, 62, 11869-11875.

[29] Sélo, I., Clément, G., Bernard, H., Chatel, J. et al., Allergy to bovine beta-lactoglobulin: specificity of human IgE to tryptic peptides. Clin. Exp. Allergy 1999, 29, 1055-1063.

[30] Mace, O. J., Marshall, F., Digestive physiology of the pig symposium: gut chemosensing and the regulation of nutrient absorption and energy supply. J. Anim. Sci. 2013, 91, 1932-1945. 


\section{Legend to Figures}

Figure 1. RP-HPLC comparison of peptides arising from the digestion of CN, SMP and WPI according to the simplified and integrate models. Chromatograms after gastro-pancreatic and gastro-pancreatic + BBM hydrolysis are reported as blue and red lines, respectively. Y, F and W indicate free Tyr, Phe and Trp, respectively. The HPLC profiles of unhydrolyzed casein and whey proteins separated in the same conditions can be found in [1].

Figure 2. Degree of hydrolysis determined at the several digestion phases (mean of three determinations). Error bars represent the standard deviation values.

Figure 3. $\beta$ - and $\alpha_{\mathrm{s} 1}$-casein peptides identified in the $\mathrm{CN}$ digesta produced with the simplified and integrated digestion models. Digesta are quite divergent, though the proteins contain specific domains that appear resistant to proteolysis regardless of the digestion procedure.

Figure 4. $\beta$-Lg peptides identified in the WPI digesta arising from the simplified and integrated digestion models. The WPI digesta share a large number of $\beta$-Lg peptides, which mostly derive from three protein domains. 
Figure 1
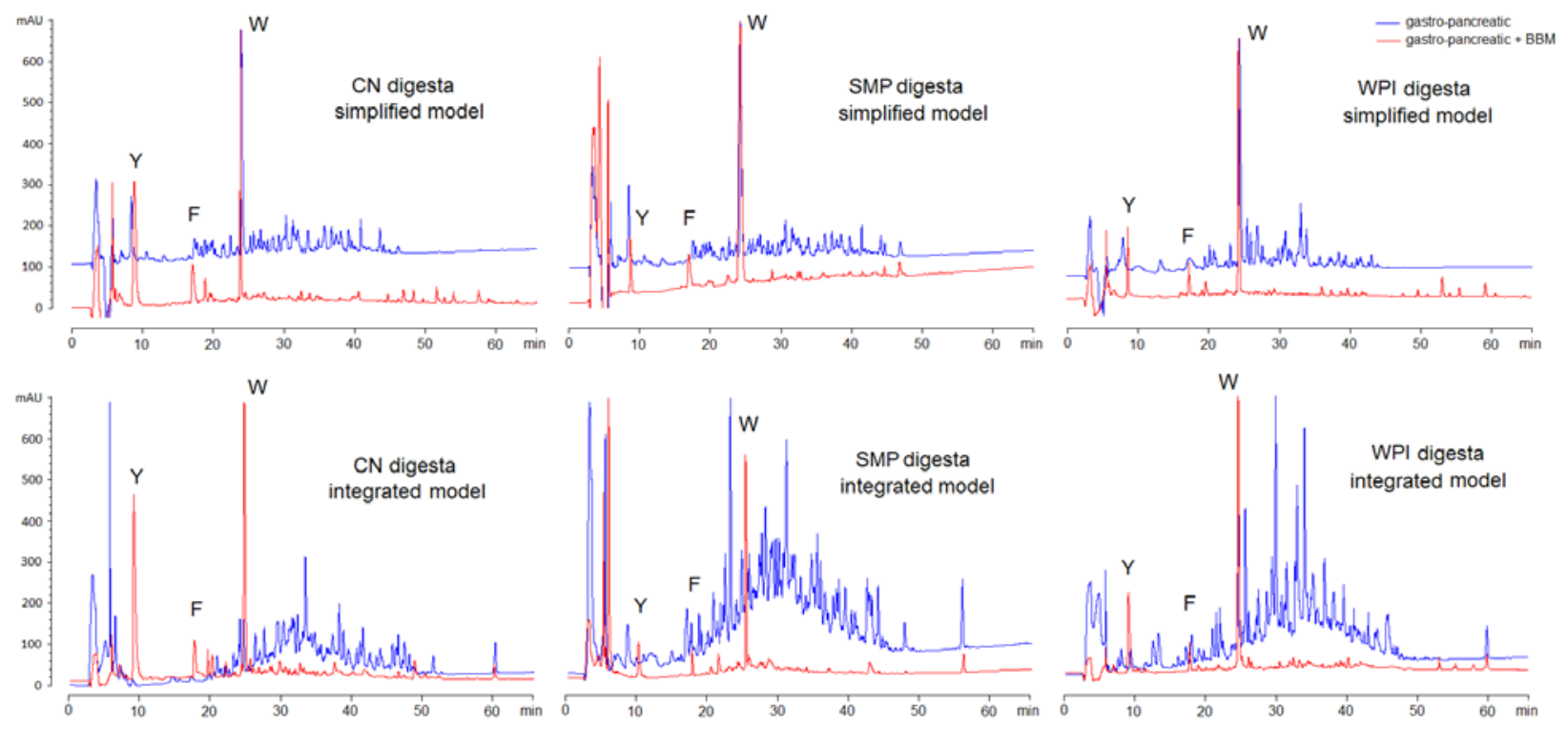

Figure 2

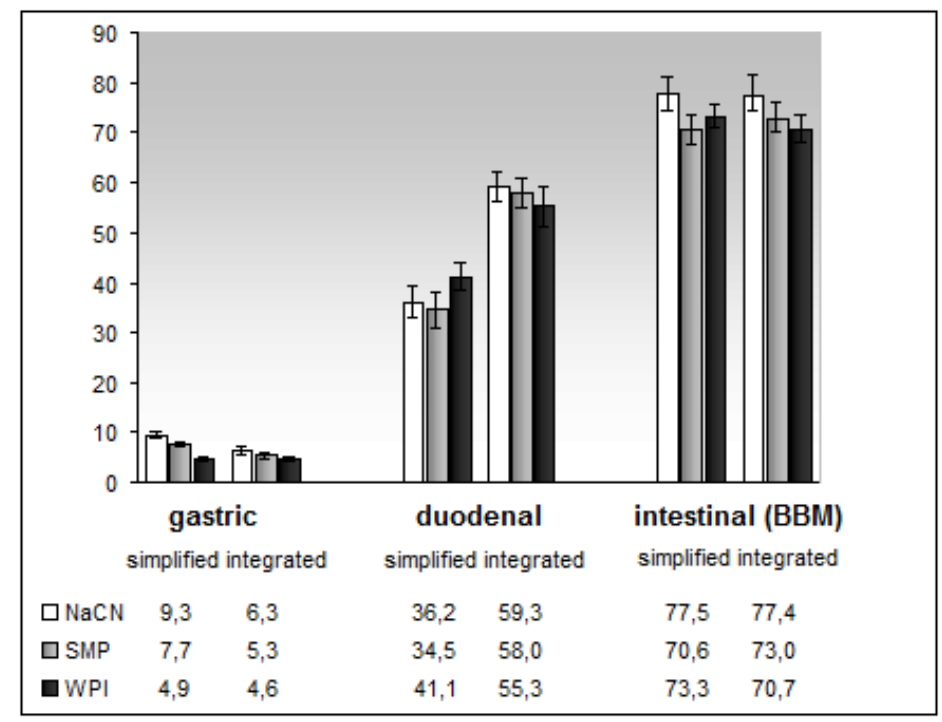


Figure 3

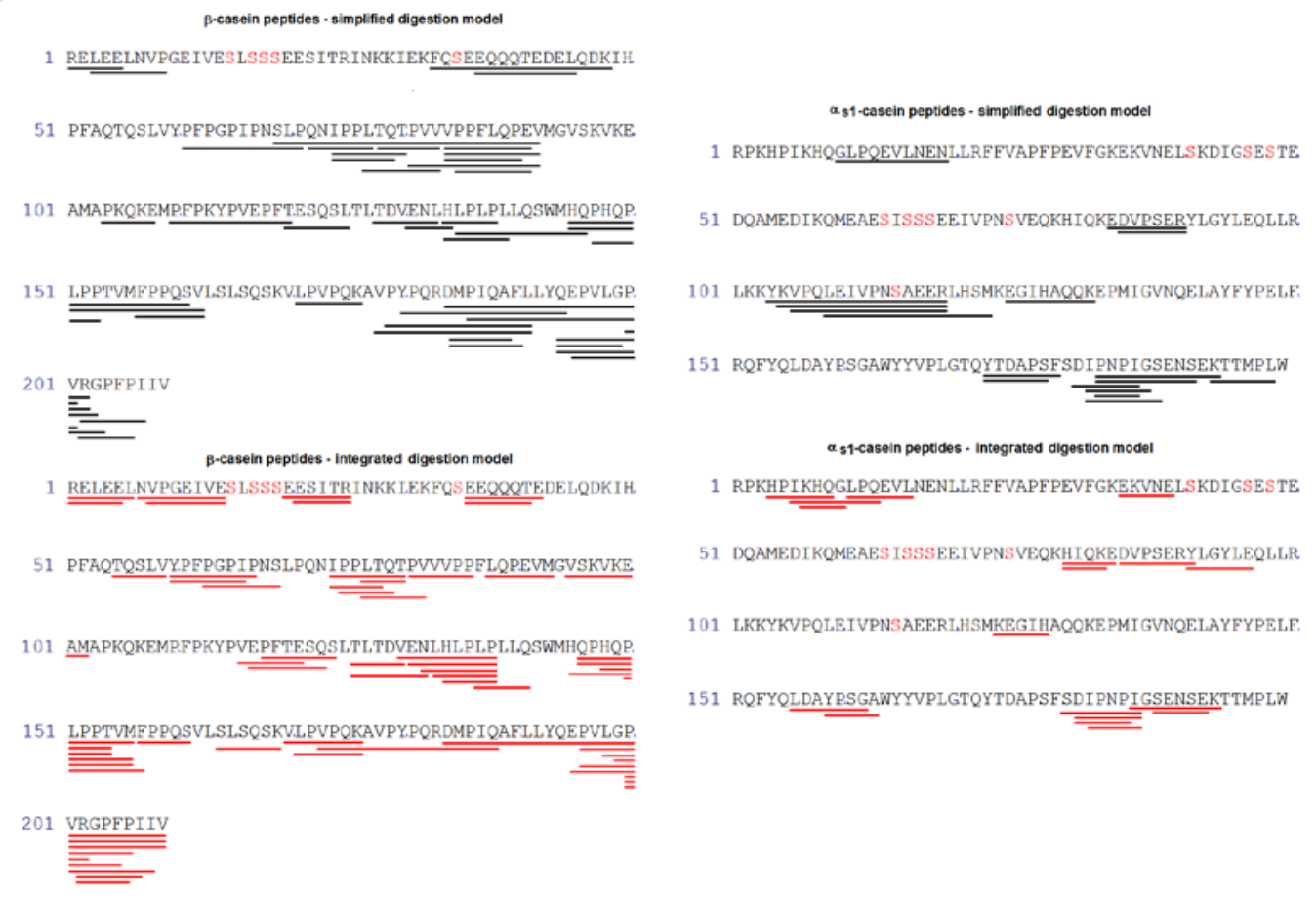

Figure 4

$\beta$-Lg peptides - simplified model

1 LIVTQTMKGLDIQKVAGTWYSLAMAASDISLLDAQSAPLRVYVEELKPTP

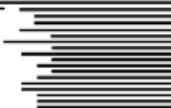

51 EGDLEILLQKWENGECAQKKI IAEKTKI PAVFKIDALNENKV WVLDTDYK

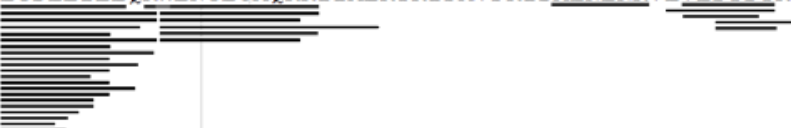

$101 \overline{\text { KYLLFCMENSAEFEQSLACQCLVRTPEVDDEALEKFDKALKALPMHIRLS }}$

151 FNPTQLEEQCHI

$\beta$-Lg peptides - integrated digestion model

1 LIVTQTMKGLDIQKVAGTWYSLAMAASDISLLDAQSAPLRVYVEELKPTP.

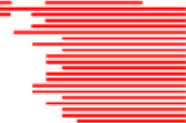

51 EGDLEILLQKWENGECAQKKI IAEKTKIPAVFKIDALNENKVLVLDTDYK

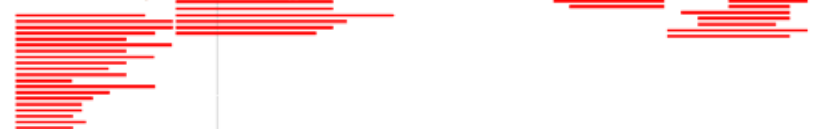

101 KYLLFCMENSAEFEQSLACQCLVRTPEVDDEALEKFDKALKALPMHIRLS

151 FNPTQLEEQCHI

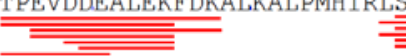

